\title{
Computed tomography (CT) scan challenges the result of SARS-CoV-2 nucleic acid test in a suspected COVID-19 case
}

\author{
Kun Yan MS ${ }^{1, a}$, Jingfeng Zhang MD, PhD ${ }^{1,2, a}$, Yangfan Zhang BS ${ }^{3}$, Shun Zhang MS ${ }^{1}$, Ting Cai MS $S^{1,2}$ and Jianjun Zheng MS ${ }^{1,2}$ \\ ${ }^{1}$ Hwa Mei Hospital, University of Chinese Academy of Sciences, Ningbo, Zhejiang, China, ${ }^{2}$ Ningbo Institute of Life and Health, University of Chinese Academy of \\ Sciences, Ningbo, Zhejiang, China and ${ }^{3}$ Ningbo University School of Medicine, Ningbo, Zhejiang, China
}

To the Editor-At present, COVID-19 is a global pandemic. The reverse-transcriptase polymerase chain reaction (RT-PCR) test is the gold standard for diagnosing COVID-19. We report a case with the false-positive result of SARS-CoV-2 nucleic acid testing in which chest computed tomography (CT) findings challenged the diagnosis of COVID-19 pneumonia. ${ }^{1,2}$

On February 16, 2020, a 36-year-old man presented to a local primary healthcare clinic with a mild cough and subjective fever for 1 day. He had no history of contact with COVID-19 patients. Physical examination revealed a body temperature of $38.4^{\circ} \mathrm{C}$. A routine blood panel showed normal ranges of white blood cells count $\left(8.9 \times 10^{9} / \mathrm{L}\right)$, neutrophils $\left(6.1 \times 10^{9} / \mathrm{L}\right)$, and lymphocytes $\left(1.8 \times 10^{9} / \mathrm{L}\right)$, as well as a high level of $\mathrm{C}$ reactive protein $(25.2$ $\mathrm{mg} / \mathrm{L})$. An oropharyngeal swab tested negative for influenza $\mathrm{A}$ and B but positive for SAR-CoV-2 by RT-PCR on February 17. The patient's chest CT showed large, mixed, ground-glass opacity (GGO) in the lower lobe of right lung with partial consolidation, distribution along with bronchovascular bundles, and a little effusion of the right oblique fissure (Fig. 1, Panel A).

On February 18, the patient was transferred to our hospital for isolation and treatment as a "confirmed case." According to a consultation among a multidisciplinary team, the diagnosis for COVID-19 seemed to be questionable according to the CT manifestations. Therefore, some imperative measures were taken as follows: (1) the patient was isolated in a single ward; (2) the same specimen was recollected as soon as possible and testing was repeated; (3) the patient's serum was tested for the virus-specific antibody of IgM for SAR-CoV-2; (4) a blood culture for bacteria or fungi was performed; and (5) an antibacterial agent was administered (moxifloxacin hydrochloride tablets, 0.4 g orally 4 times

per day) as well as an antiviral agent (lopinavir/ritonavir tablets 400 $\mathrm{mg} / 100 \mathrm{mg}$ orally twice daily).

The patient's serum tested negative for SARS-CoV-2-specific IgM antibody on February 18. Consecutive samples were collected for SARS-CoV-2 testing daily from February 18 to February 20 (ie, oropharyngeal swab and sputum). All of the repeated tests of viral nucleic acid were negative. A 5-day blood culture demonstrated no growth of bacteria or fungi. The patient's symptoms gradually improved. A repeat chest CT scan on February 21 showed that the lesions in the right lower lobe had been partially absorbed (Fig. 1, panel B).

On February 22, bronchoalveolar lavage fluid was collected for SARS-CoV-2 testing by RT-PCR and the result was also negative. On February 24, the patient's serum tested negative for SARSCoV-2-specific IgG antibody. All of his symptoms had disappeared by February 26. A follow-up chest CT on February 29 showed that the lesions in the right lower lobe had completely resolved (Fig. 1, panel C). After a consultation with the COVID19 prevention and control committee in our hospital, the diagnosis was revised to community-acquired pneumonia (CAP) considering no history of contact with COVID-19 patients and the negative results of consecutive repeat RT-PCR tests and the serum antibody test. The patient was released from quarantine and was discharged on the same day.

Yan et $\mathrm{al}^{3}$ reported that failing to consider COVID-19 because of a positive rapid test result for dengue fever, which has serious implications not only for the patient but also for public health. (a)

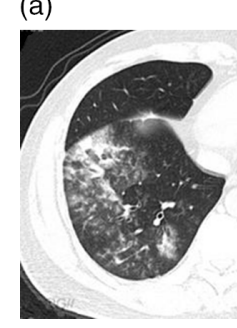

(b)

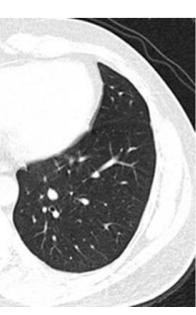

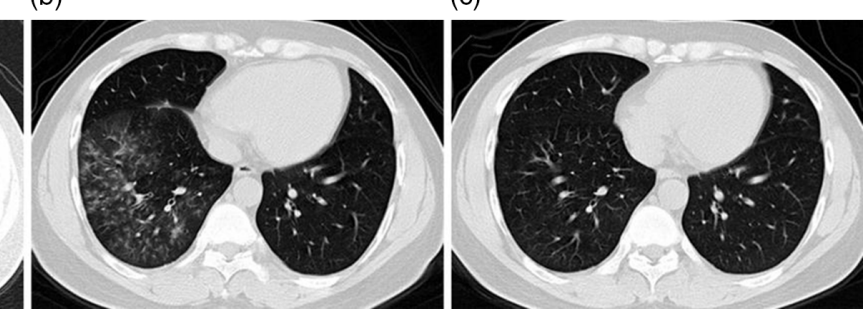

Fig. 1. Chest computed tomography (CT) images of a 36-year-old man with a false-positive nucleic acid test result for SARS-CoV-2. The revised diagnosis was community-acquired pneumonia. Panel A: chest CT obtained on February 16 shows large, mixed, ground-glass opacity (GGO) in the right lower lobe with partial consolidation, distribution along with

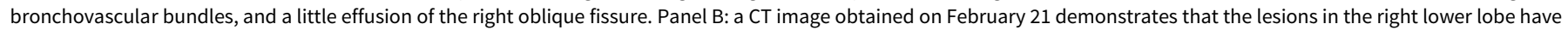
been partially absorbed. Panel C: a CT image obtained on February 29 shows that the lesions in the lower right lobe have completely resolved.

Author for correspondence: Jianjun Zheng, E-mail: zhengjianjun@ucas.ac.cn

a Authors of equal contribution.

Cite this article: Yan K, et al. (2020). Computed tomography (CT) scan challenges the result of SARS-CoV-2 nucleic acid test in a suspected COVID-19 case. Infection Control \& Hospital Epidemiology, 41: 1362-1363, https://doi.org/10.1017/ice.2020.171

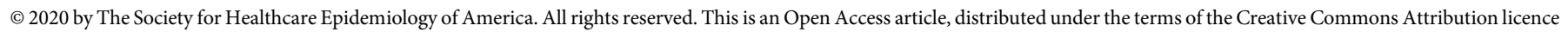
(http://creativecommons.org/licenses/by/4.0/), which permits unrestricted re-use, distribution, and reproduction in any medium, provided the original work is properly cited. 
Our case highlights the importance of timely recognition of a falsepositive result for SARS-CoV-2 when chest CT findings do not conform to the typical changes of COVID-19 pneumonia. From the epidemic control perspective, it is imperative to isolate individuals with suspected cases of COVID-19 to protect the uninfected population. However, the suspected cases should not be housed with confirmed cases of COVID-19 to prevent the spread of this disease.

Acknowledgments. We are appreciated with Dr.Chuyi Cai and Prof. Getu Zhaori for their kind help to translate and polish some contents of the manuscript.

Financial support. This work was supported by Key Research Foundation of Hwa Mei Hospital, University of Chinese Academy of Sciences, China (Grant No. 2020HMZD19, 2020HMZD20).
Conflicts of interest. All authors report no conflicts of interest relevant to this article.

\section{References}

1. Bernheim A, Mei X, Huang M, et al. Chest CT findings in coronavirus disease-19 (COVID-19): relationship to duration of infection. Radiology February 20, 2020 [Epub ahead of print]. doi: 10.1148/radiol.2020 200463.

2. Chua F, James DA, Desai SR, et al. The role of CT in case ascertainment and management of COVID-19 pneumonia in the UK: insights from highincidence regions. Lancet Infect Dis March 25, 2020 [Epub ahead of print]. doi: 10.1016/S2213-2600(20)30132-6.

3. Yan G, Lee CK, Lam LTM, et al. Covert COVID-19 and false-positive dengue serology in Singapore. Lancet Infect Dis 2020 March 4 [Epub ahead of print]. doi: 10.1016/S1473-3099(20)30158-4.

\title{
A dynamic residential community-based quarantine strategy: China's experience in fighting COVID-19
}

\author{
Yan Guo PhD ${ }^{1,2}$, Yiran Li MS², Aliza Monroe-Wise MD, MSc 3 , Sai-Ching Jim Yeung MD, $\mathrm{PhD}^{4}$ and Yixiang Huang PhD ${ }^{5,1}$ (i) \\ ${ }^{1}$ Sun Yat-sen Global Health Institute, Sun Yat-sen University, Guangzhou, China, ${ }^{2}$ Department of Medical Statistics, School of Public Health, Sun Yat-sen \\ University, Guangzhou, China, ${ }^{3}$ Department of Global Health, University of Washington, Seattle, Washington, United States, ${ }^{4}$ Department of Emergency Medicine, \\ The University of Texas MD Anderson Cancer Center, Houston, Texas, United States and ${ }^{5}$ Department of Health Policy and Management, School of Public Health, \\ Sun Yat-sen University, Guangzhou, China
}

To the Editor-As the global COVID-19 pandemic progresses, many countries face major public health emergencies. The number of active COVID-19 cases worldwide is $>1.69$ million as of April $12,2020 .{ }^{1} \mathrm{~W}$ ith rapidly increasing new cases, healthcare systems are at the brink of collapse in some regions. To reduce the burden on health systems, public health strategies should be adopted to control the source of infection, to cut off transmission routes, and to protect vulnerable populations. One strategy that was effective in China in controlling the COVID-19 epidemic was the successful implementation of a nationwide, community-based, dynamic quarantine strategy. ${ }^{2}$ From Late January to March 18, 2020, the main purpose was to prevent COVID-19 from spreading in China. Afterward, the focus turned to the prevention of imported cases.

At the beginning of China's quarantine, residents were required to stay home. When necessary, they were required to use an electronic pass system with traceable personal information to gain entry to residential areas, work places, and public transportation, and body temperature was screened by thermal scanning at the entrances. ${ }^{3}$ Once new cases were identified, health professionals and volunteers followed-up, treated, and isolated the patient and those in close contact. From prior experience with SARS, most Chinese citizens understood the importance of the quarantine and were invested in its success. As the epidemic came under better control, community-level management was strengthened and upgraded. It also became more dynamic, allowing movement

Author for correspondence: Yixiang Huang, E-mail: huangyx@mail.sysu.edu.cn

Cite this article: Guo Y, et al. (2020). A dynamic residential community-based quarantine strategy: China's experience in fighting COVID-19. Infection Control \& Hospital Epidemiology, 41: 1363-1364, https://doi.org/10.1017/ice.2020.172 and return to work to minimize the effects on people's lives and businesses while still monitoring movements and health status. ${ }^{4}$

Experience can be drawn from China's quarantine strategy. First, the quarantine strategy was strictly implemented nationwide at a community level. ${ }^{5}$ Strong governmental support is required to strengthen the community, and training is needed to foster policy implementation. Second, the quarantine strategy was dynamic and was adjustable based on the evolving situations, from stay-home quarantine to movement with updated health monitoring. Third, a large team of professional and technical support traced, identified, treated, and isolated patients and their close contacts. These strategies ensured early diagnosis and treatment, thus bringing the COVID-19 pandemic under control in China.

As the COVID-19 pandemic continues to take a massive toll on the healthcare systems and economies of countries worldwide, China's experience in its fight against this novel virus can be of great value to other countries. A practical attitude of learning by doing and responsiveness among government officials and the general population must be fostered.

\section{Acknowledgments.}

Financial support. This work was supported by the National Social Science Fund of China (grant no. 18BGL218) and the China Medical Board (grant no. 17-271).

Conflicts of interest. All the authors declare no conflicts of interest.

\section{References}

1. Coronavirus disease 2019 (COVID-19) situation report, 2020. World Health Organization website. https:/www.who.int/emergencies/diseases/novelcoronavirus-2019/situation-reports. Published 2020. Accessed April 13, 2020. 